\title{
Use of host- and bacteria-derived salivary markers in detection of periodontitis: A cumulative approach
}

\author{
Ulvi Kahraman Gursoya,b,c,*, Eija Könönen ${ }^{a, b}$, Pirkko J. Pussinen ${ }^{c}$, Taina Tervahartiala ${ }^{c}$, \\ Kati Hyvärinen ${ }^{c}$, Anna L. Suominen ${ }^{\mathrm{a}, \mathrm{d}}$, Veli-Jukka Uitto ${ }^{\mathrm{c}}$, Susanna Paju ${ }^{\mathrm{c}, \mathrm{e}}$ and Timo Sorsa ${ }^{\mathrm{c}}$ \\ anstitute of Dentistry, University of Turku, Turku, Finland \\ ${ }^{\mathrm{b}}$ Department of Infectious Disease Surveillance and Control, National Institute for Health and Welfare (THL), \\ Helsinki, Finland \\ ${ }^{\mathrm{c}}$ Institute of Dentistry, University of Helsinki, and Department of Oral and Maxillofacial Surgery, Helsinki \\ University Central Hospital, Helsinki, Finland \\ ${ }^{\mathrm{d}}$ Unit of Living Conditions, Health and Wellbeing, and Department of Environmental Health in Environmental \\ Epidemiology Unit, National Institute for Health and Welfare (THL), Kuopio, Finland \\ e Unit for Specialized Oral Care in the Metropolitan Area and Kirkkonummi, Helsinki, Finland
}

\begin{abstract}
In the present study, we propose a novel diagnostic approach, using 3 different salivary markers, representing periodontal pathogen burden, inflammation, and tissue degradation, for detecting periodontitis. The salivary concentrations of Porphyromonas gingivalis, interleukin- $1 \beta$, and matrix metalloproteinase- 8 , available from salivary specimens of 165 subjects (84 subjects with advanced periodontitis and 81 controls), were calculated together to obtain a cumulative risk score (CRS). In the calculation of CRS, the concentrations of each marker were divided into tertiles, and cumulative sub-score per each subject were calculated by the multiplication of the tertile values. Three CRS groups, indicating the lowest, medium, or highest risk, were formed with the cumulative sub-scores. Logistic regression analysis and ROC curves were performed to study the association of CRS with periodontitis. The results indicate that CRS, calculated from the 3 salivary biomarkers, is associated with advanced periodontitis more strongly than any of the markers individually. CRS offers a novel, non-invasive model for advanced periodontitis risk categorization that is especially useful in large population surveys where a periodontal examination is not feasible.
\end{abstract}

Keywords: IL-1 $\beta$, MMP-8, Porphyromonas gingivalis, risk score, saliva

\section{Introduction}

Periodontitis forms a group of infection-induced inflammatory diseases, initiated by imbalance between pathogenic microorganisms in subgingival biofilm, affecting tooth-supporting soft tissues and alveolar bone [1]. The traditional diagnosis of periodontitis is based on clinical and radiographic assessments of periodontal tissues. However, when assessing clinically the

* Address for correspondence: Ulvi Kahraman Gursoy, Institute of Dentistry, Lemminkäisenkatu 2, University of Turku, 20520, Turku, Finland. Tel.: +358 40 4194735; E-mail: ulvi.gursoy@utu.fi. periodontal health status in large-scale health surveys, a full periodontal examination turns to be laborious and expensive, requiring trained dental professionals [2].

The most desirable goal in health care promotion and delivery would be to monitor the periodontal health status and the onset, progression, and treatment outcome of periodontal diseases through non-invasive means. As an easily collected and non-invasive specimen, saliva has been used as a diagnostic fluid in medicine [3, 4]. Elevated levels of bacteria, bacterial products (lipopolysaccharide; LPS, enzymes), host-originated enzymes, cytokines, and bone turnover biomarkers have been found in saliva of periodontitis patients in relation to their controls $[3,4]$. 


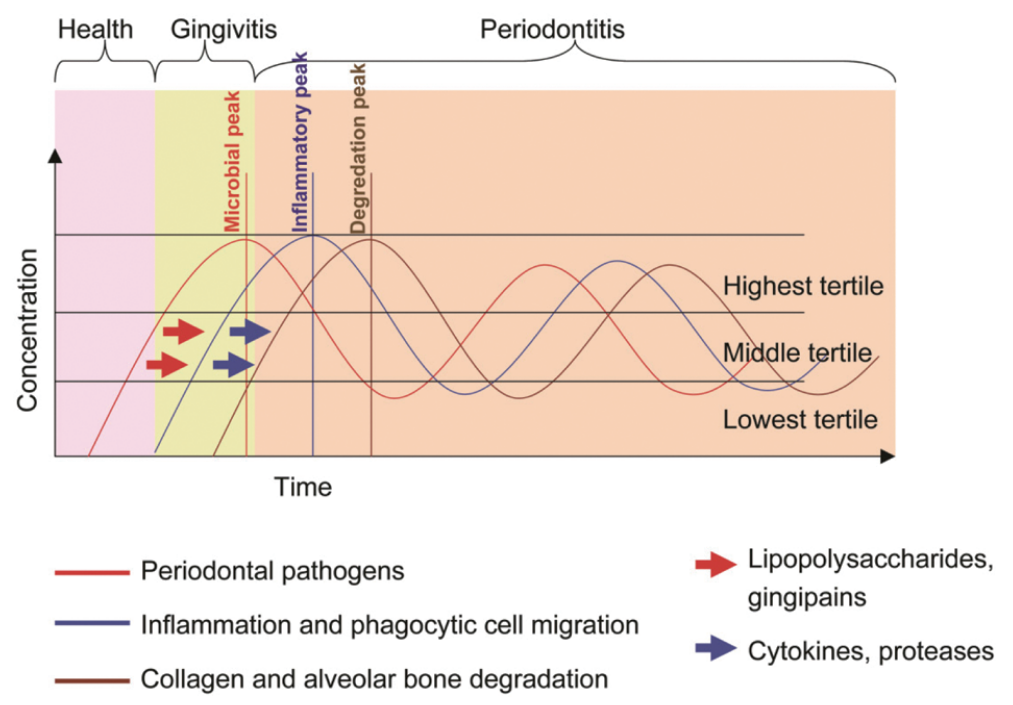

Fig. 1. A schematic presentation of the episodic character of chronic periodontitis. With the increase in microbial amounts, bacterial lipopolysaccharides and gingipains, the secretion proinflammatory cytokines are secreted from epithelial cells and fibroblasts. Cytokine and protease levels increase, as leukocytes migrate towards the site of infection. The increase in cytokine levels stimulates the osteoclastogenesis via increasing the Receptor Activator for Nuclear Factor $\kappa \mathrm{B}$ Ligand and decreasing the osteoprotegerin levels. Consecutively, host proteases degrade collagens in connective and bone tissues.

During the onset of periodontitis, markers of inflammation, such as interleukin (IL)-1 $\beta$, IL-6, and tumor necrosis factor (TNF)- $\alpha$, are released from cells of the junctional epithelium. In the course of the disease, a number of enzymes, such as matrix metalloproteinase (MMP)-8, MMP-9, and MMP-13, are produced and activated by host cells in a cascade leading to degradation of tooth-supporting soft and bone tissues [1,5, 6]. As a result of bone degradation, pyridinoline crosslinked carboxyterminal telopeptide (ICTP) and osteocalcin are released into periodontal tissues and, eventually, into saliva [3,7-11]. Periodontal pathogens initiate the inflammatory conditions; hence, their simple carriage or presence in high numbers in saliva have been associated with periodontitis [12-18].

Various oral bacteria, their by-products, cytokines, host enzymes, and biomarkers of bone turnover have been analyzed from saliva aiming to find a valuable marker in distinguishing the subjects with periodontitis and periodontally healthy subjects $[3,4]$. When searching a potential salivary biomarker for detecting periodontitis, a common approach in cross-sectional studies is to look for a statistical difference $(p<0.05)$ between periodontitis and control subjects and to determine a meaningful threshold for the tested marker. So far, this aim has not been fulfilled. Many inconsistencies in the current literature are generally attributed to the heterogeneity of detection techniques as well as local and systemic factors that may affect the detection level of examined markers in saliva.

Our hypothesis is that the increase or the decrease in bacterial burden, inflammatory response, and tissue destruction do not occur simultaneously but consecutively at different sites of periodontal tissues (Fig. 1). Bacterial LPS and enzymes, and cytokines and enzymes produced by host cells work as the major determinants of this cascade. When bone resorption is at its highest level due to the increased stimulation of osteoclastogenesis by inflammatory cytokines and neutrophilic enzymes, the same response suppresses the level of bacterial burden. This means that a bacteria-derived biomarker can be present at a low level, while bone resorption is at its highest level. Also, a biomarker responsible for bone degradation may fail to be connected to the disease at a phase when the microbial attack is at its highest level.

In the present study, we used clinical, microbiological, and inflammatory data available from our recent studies $[7,13,14]$ and propose a novel statistical approach for diagnosing periodontitis to be used, in particular, in public health surveys or in large crosssectional studies. In this approach, the salivary concentrations of selected biomarkers, representing periodontal pathogen burden, inflammation, and tissue degradation, have been combined to obtain a cumulative risk score (CRS). 
Table 1

Study population by the salivary biomarkers, including the concentrations of periodontal pathogens, IL-1 $\beta$, and enzymes examined (GE: Genome equivalent). Data come from our published studies [7,13,14]

\begin{tabular}{|c|c|c|c|c|}
\hline & $\begin{array}{l}\text { No. of subjects negative/ } \\
\text { positive for markers }\end{array}$ & Periodontitis $(n=84)$ & Control $(n=81)$ & $\mathrm{p}$ value \\
\hline A. actinomycetemcomitans $(\mathrm{GE} / \mathrm{ml})($ mean $\pm \mathrm{SD})$ & $108 / 57$ & $1 \times 10^{5} \pm 6 \times 10^{5}$ & $1 \times 10^{4} \pm 3 \times 10^{4}$ & 0.009 \\
\hline P. gingivalis $(\mathrm{GE} / \mathrm{ml})($ mean $\pm \mathrm{SD})$ & $53 / 112$ & $2 \times 10^{6} \pm 8 \times 10^{6}$ & $2 \times 10^{4} \pm 8 \times 10^{4}$ & $<0.001$ \\
\hline P. intermedia $(\mathrm{GE} / \mathrm{ml})($ mean $\pm \mathrm{SD})$ & $104 / 61$ & $1 \times 10^{8} \pm 3 \times 10^{8}$ & $3 \times 10^{6} \pm 1 \times 10^{7}$ & $<0.001$ \\
\hline T. denticola $(\mathrm{GE} / \mathrm{ml})($ mean $\pm \mathrm{SD})$ & $96 / 69$ & $1 \times 10^{7} \pm 1 \times 10^{7}$ & $2 \times 10^{6} \pm 1 \times 10^{7}$ & 0.012 \\
\hline T. forsythia $(\mathrm{GE} / \mathrm{ml})($ mean $\pm \mathrm{SD})$ & $31 / 134$ & $5 \times 10^{7} \pm 9 \times 10^{5}$ & $1 \times 10^{7} \pm 4 \times 10^{7}$ & $<0.001$ \\
\hline $\mathrm{IL}-1 \beta(\mathrm{pg} / \mathrm{ml})($ mean $\pm \mathrm{SD})$ & $0 / 165$ & $665.7 \pm 267.6$ & $467.8 \pm 279.9$ & $<0.001$ \\
\hline MMP-8 (ng/ml) $($ mean \pm SD $)$ & $0 / 165$ & $1000.5 \pm 717.7$ & $450.9 \pm 420.4$ & $<0.001$ \\
\hline TIMP-1 (ng/ml) (mean \pm SD) & $2 / 163$ & $104.7 \pm 112.6$ & $136.0 \pm 99.7$ & 0.001 \\
\hline $\operatorname{ICTP}(\mathrm{ng} / \mathrm{ml})($ mean $\pm \mathrm{SD})$ & $0 / 165$ & $0.95 \pm 0.97$ & $0.74 \pm 0.49$ & 0.039 \\
\hline
\end{tabular}

\section{Materials and methods}

\subsection{Data collection}

The present study population is a subsample of the nationally representative population of the "Health 2000 Health Examination Survey". A total of 8,028 subjects, aged $\geqslant 30$ years and steadily living in Finland, participated in the health examination in the field, divided in 5 districts, where data on their general and oral health were collected. Periodontal measurements from 4 sites per tooth included probing pocket depths (PPD) and bleeding on probing. Each tooth was recorded as having PPD $<4 \mathrm{~mm}, \mathrm{PPD} \geqslant 4 \mathrm{~mm}$, or PPD $\geqslant 6 \mathrm{~mm}$. Paraffin-stimulated whole saliva samples were collected from 1,294 of the Health 2000 subjects in the southern district, and frozen at $-70^{\circ} \mathrm{C}$ until further used [12]. Information about data collection methods can be found in the report of the National Public Health Institute (http://www.terveys2000.fi/julkaisut/oral_health.pdf). All protocols had been approved by the institutional ethics committees.

Salivary data used in the present analysis were obtained from a subpopulation of 165 subjects, divided into 2 extreme groups on the basis of their periodontal status; 84 advanced periodontitis subjects with at least 14 teeth with PPD of $\geqslant 4 \mathrm{~mm}$ (mean age 49.6 years) and 81 control subjects with no pocket teeth (mean age 47.9 years). All subjects had at least 20 teeth. The detailed information on the techniques used for the salivary analyses of periodontal pathogen burden and inflammatory and tissue destruction markers can be found elsewhere [7,13,14]. Briefly, frozen saliva samples were thawed and centrifuged, and the supernatants were aliquoted. The salivary concentrations of the 3 markers used in the present study were detected as follows: IL- $1 \beta$ with a commercial ELISA kit (Amersham, GE Healthcare, Buckinghamshire, UK), MMP-8 with a time-resolved immunofluorometric assay (IFMA), and Porphyromonas gingivalis with a quantitative real-time PCR assay [7,13,14].

\subsection{Selection of the biomarkers for calculating $C R S$}

On the basis of the association with periodontitis, we previously concluded that the salivary concentrations of IL- $1 \beta$, MMP- 8 , tissue inhibitor of matrix metalloproteinase (TIMP) -1, and ICTP, and the presence of pathogenic species in saliva could potentially be used as markers of periodontitis (Table 1) [7,13,14]. From the examined host- and bacteria-derived markers, $P$. gingivalis, IL-1 $\beta$, and MMP- 8 were selected as the most potential markers of periodontal pathogen burden, inflammation, and tissue destruction, respectively, for present analyses.

\subsection{Calculation of cumulative risk scores $(C R S)$}

For the calculation of CRS, the salivary concentrations of $P$. gingivalis, IL-1 $\beta$, and MMP-8, which were separately analyzed in our previous studies $[7,13,14]$, were statistically re-analyzed with a novel approach as follows.

The salivary concentrations of each marker, originally collected as continuous data, were divided into tertiles 1-3 (Table 2). A cumulative sub-score per each subject was calculated by the multiplication of the corresponding tertile values of $P$. gingivalis, IL- $1 \beta$, and MMP-8. According to this calculation, the subject's cumulative sub-score can be $1,2,3,4,8,9,12,18$, or 27. Furthermore, based on these cumulative subscores, 3 CRS groups were formed as follows:

CRS I: Lowest risk (the cumulative sub-scores of 1 , $2,3)$

CRS II: Medium risk (the cumulative sub-scores of $4,8,9)$ 
Table 2

Tertile values of $P$. gingivalis, IL-1 $\beta$, and MMP-8 were calculated from the salivary concentrations of each marker that were originally collected as continuous data

\begin{tabular}{|c|c|c|c|c|c|c|}
\hline \multirow{3}{*}{ Tertile value } & \multicolumn{6}{|c|}{ Range } \\
\hline & \multicolumn{2}{|c|}{ P. gingivalis $(\mathrm{GE} / \mathrm{ml})$} & \multicolumn{2}{|c|}{$\mathrm{IL}-1 \beta(\mathrm{pg} / \mathrm{ml})$} & \multicolumn{2}{|c|}{ MMP-8 (ng/ml) } \\
\hline & minimum & maximum & minimum & maximum & minimum & maximum \\
\hline 1 & 0.00 & 387.2 & 35.14 & 411.9 & 2.8 & 315.8 \\
\hline 2 & 434.5 & $18,764.4$ & 419.8 & 685.1 & 318.0 & 780.6 \\
\hline 3 & 19,980 & $51,373,537.5$ & 716.9 & 1260.1 & 785.3 & $2,576.6$ \\
\hline
\end{tabular}

CRS III: Highest risk (the cumulative sub-scores of $12,18,27)$

For example, a subject with salivary concentrations of $550 \mathrm{GE} / \mathrm{ml}$ for $P$. gingivalis, $600 \mathrm{pg} / \mathrm{ml}$ for IL- $1 \beta$, and $300 \mathrm{ng} / \mathrm{ml}$ for MMP-8 will have tertile values of 2 , 2 , and 1 , correspondingly (Table 2 ). When these tertile values are multiplied $(2 \times 2 \times 1)$, a cumulative subscore will be 4 , and the subject will be categorized as being at medium risk of having periodontitis (CRS II).

\subsection{Statistical analysis}

The SPSS statistical program (Version 15.0; SPSS Inc., Chicago, IL, USA) was used to analyze the data. An ANOVA test was used to compare the biomarker concentrations between the periodontitis and control groups. A logistic regression analysis and receiver operating characteristics (ROC) curves were performed to study the association of $P$. gingivalis, IL-1 $\beta$, MMP-8, and CRS as tertiles with periodontitis.

\section{Results}

For the calculation of CRS, the salivary concentrations of $P$. gingivalis, IL- $1 \beta$, and MMP- 8 were selected due to their high prevalence in the population and significantly different levels between periodontitis and control subjects. The reasons to exclude the other markers (Table 1) were as follows: the low prevalence of Aggregatibacter actinomycetemcomitans, Treponema denticola, and Prevotella intermedia among the study population, the weak statistical significance of ICTP values between the periodontitis and control groups and the lacking diagnostic contribution of Tannerella forsythia and TIMP-1 to the present model.

Table 3 presents the odds ratios and area under curve (AUC) values with $95 \%$ confidence intervals separately for each of the 3 selected biomarkers and cumulatively as CRS. Each marker had an association with periodontitis at a different level. The association increased considerably by using CRS, which had 2 to
3 times higher odds ratios in comparison with single biomarkers. The increase in the diagnostic accuracy was also demonstrated in the ROC analyses, where the AUC value increased from $0.694-0.710$ up to 0.766 .

\section{Discussion}

The present study proposes a novel periodontitis risk categorization model, taking into account periodontal pathogen, inflammation, and tissue degradation markers in saliva. The strength of this new approach is that when one of the 3 biomarkers, e.g., the amount of periodontopathogenic bacteria, is in remission due to the strong host response, the concentration of other 2 biomarkers, associated with inflammation and tissue degradation, will be exacerbated, but CRS will still identify the subjects with the disease. This will diminish the number of false negatives which are often obtained in single-marker analyses.

Even after 3 decades of work, there is not yet a clear consensus on the strength of associations between salivary biomarkers and periodontal diseases. A good example of this controversy is the detection of pathogenic bacteria in saliva and its association with periodontitis. According to Paju et al. [15], who analyzed the saliva samples of 1198 adult dentate subjects, the number of pathogenic species rather than a certain periodontal pathogen is associated with periodontitis. In contrast, Leblebicioglu et al. [16] concluded that the presence of periodontal pathogens in saliva correlates with signs of gingivitis but not with alveolar bone loss (periodontitis). Interestingly, in a study by Sawamoto et al. [17], salivary A. actinomycetemcomitans levels proved to be higher in periodontally healthy subjects than in periodontitis patients. Hamlet et al. [18] suggested that the deepened pocket depth, a clinical marker of periodontitis, is associated with the presence of $P$. gingivalis and $A$. actinomycetemcomitans but not with that of $P$. intermedia. However, in a more recent study by Könönen et al. [12], the number of teeth with pocket depths of $\geqslant$ $4 \mathrm{~mm}$ was associated with the salivary detection rates 
Table 3

Odds ratios (OR) and area under curve (AUC) values (95\% confidence interval) indicating the association between the presence of periodontitis and the 3 salivary biomarkers divided into tertiles. The associations were also calculated for cumulative risk scores (CRS). In the risk group column, III represents the highest tertile, II the medium tertile, while I represents the lowest tertile

\begin{tabular}{lcccc}
\hline & Risk group & $\begin{array}{c}\text { No. of periodontitis/ } \\
\text { control subjects }\end{array}$ & OR (95\% CI) & AUC (95\% CI) \\
\hline P. gingivalis & III & $43 / 12$ & $8.01(3.39-18.89)$ & $0.710(0.631-0.789)$ \\
& II & $24 / 31$ & $1.7(0.7-3.7)$ & \\
IL-1 $\beta$ & I & $17 / 38$ & 1 & \\
& III & $38 / 17$ & $6.54(2.8-15.06)$ & $0.694(0.613-0.775)$ \\
MMP-8 & II & $32 / 23$ & $4.07(1.81-9.15)$ & \\
& I & $14 / 41$ & 1 & \\
CRS & III & $41 / 14$ & $7.81(3.34-18.24)$ & $0.710(0.631-0.789)$ \\
& II & $28 / 27$ & $2.76(1.24-6.12)$ & \\
& I & $15 / 40$ & 1 & \\
& III & $38 / 7$ & $19.9(7.11-55.65)$ & $0.766(0.694-0.839)$ \\
& II & $34 / 30$ & $4.7(1.26-12.31)$ & \\
\hline
\end{tabular}

of $P$. gingivalis, $P$. intermedia, T. denticola, and A. actinomycetemcomitans. Such differences in the results can also be observed in studies where various cytokines have been analyzed from saliva. For example, salivary IL- $1 \beta$, a proinflammatory cytokine predominately secreted by neutrophils and macrophages, has been either associated with periodontitis [3,14], or not [19]. Similarly, higher levels of TNF- $\alpha$ have been found in salivary samples of periodontitis subjects than in those of periodontally healthy controls [20], while other researchers have failed to show such a difference [14, 21]. Usually, these discrepancies have been explained by differences in the study population, such as the age range, percentage of smoking subjects, or number of study subjects. For example, cigarette smoke inhibits the enzyme activity due to the interaction with aldehydes in smoke and host-derived enzymes [22]. Also, the detection method used has influence on the outcome; when analyzing MMP-8 from saliva, mouthrinse, or gingival crevicular fluid, IFMA results in a more reliable distinction of periodontitis subjects from their controls than by ELISA with different antibodies [7,23, 24].

It is possible that the episodic character of periodontitis plays a central role in the level of salivary biomarkers. Chronic periodontitis, the most prevalent form of destructive periodontal disease, has dynamic states of exacerbation and remission [25,26]. It is usually related to a polymicrobial infection, $T$. denticola, $P$. gingivalis, and T. forsythia being considered the major players [27]. With the increase in bacterial numbers and their virulence factors, the local inflammatory reaction in periodontal tissues activates the immune system [28]. The initial inflammatory response aims to limit bacterial invasion; hence, it promotes the infiltration of neutrophils and macrophages to the site of infection. This infiltration is achieved by the secretion of proinflammatory cytokines from epithelial cells and fibroblasts. After their migration to inflamed tissues, leukocytes suppress the bacterial invasion by their oxygen-dependent or -independent mechanisms. Subsequently, T- and B-cells emerge to the sites of infection and secrete immunoglobulins as an antigen-specific response [29,30]. While the host defense suppresses the level of infection by eliminating pathogens, the increased expression of proinflammatory cytokines, such as IL-1, -6, -11, and -17, and TNF- $\alpha$, stimulates alveolar bone resorption. These cytokines can induce osteoclastogenesis by increasing the expression of Receptor Activator for Nuclear Factor $\kappa$ B Ligand (RANKL) and by decreasing the osteoprotegerin (OPG) production in osteoblasts/stromal cells [31,32]. Matrix metalloproteinases, especially MMP-8 and -13 , can contribute to the resorption of alveolar bone. With decreasing bacterial challenge, anti-inflammatory cytokines, such as IL-4, -10, -12, -13, and -18, and lipid mediators inhibit the inflammation in periodontal tissues and bone resorption [32].

Salivary diagnostics in periodontology is not anymore trying to find a silver bullet in detection of periodontitis [33]. Therefore, the presence of multiple periodontopathogenic bacteria $[13,15]$, combinations and proportions of host-derived biomarkers [7], or combinations of host-derived biomarkers with periodontal pathogens [34] have been introduced as potential approaches for newly developed point-of-care tests in diagnosing oral and systemic diseases [6,23,24]. In above-mentioned studies, a combinational value has 
been calculated as probabilities in a logistic regression model, whereas the present CRS was calculated by multiplying the tertile values of each marker. One practical improvement of the present proposal is to define universal reference ranges (high, medium, low) for the salivary levels of each biomarker. In that way CRS would be comparable between different study populations.

In the current model, the selection of the 3 salivary markers, $P$. gingivalis, IL-1 $\beta$, and MMP-8, can be taken as an example. The major obstacle in salivary diagnostics is that several markers of periodontitis, such as TNF- $\alpha$, IL-6, RANKL, ICTP, or OPG, are found at very low levels in saliva where they are highly diluted unlike in gingival crevicular fluid. This fact limits the comparisons between periodontitis and control subjects. With the improvement of salivary detection techniques, for example, with new proteomic analyses, various bacterial, inflammatory, or tissue degradation markers can be applied in the current model. In the present study, however, the selection of the 3 disease markers is still unique, since $P$. gingivalis can efficiently process and activate IL- $1 \beta$ and MMP- 8 , thus inducing the bacteria-host destruction cascade in periodontitis [34-36]. P. gingivalis can directly stimulate collagen degradation via activation of pro-MMP- 8 by cleaving the "prodomain" with their gingipains; however, in the pathogenesis of periodontitis, the significance of this effect is not yet clarified.

As saliva is a pooled fluid of the whole mouth, the present approach is not suitable for gingival crevicular fluid. It is notable that salivary diagnostics basically aims to detect subjects with periodontitis but not to localize the disease in the dentition. Therefore, our present approach uses multiplication to result in a cumulative effect of several individual markers, even when present at low levels in saliva. The strength of CRS is in its potential in public health surveys or large cross-sectional studies with different age groups. In health care promotion and delivery in periodontics, the demonstrated potential of saliva enables to monitor the periodontal health status through non-invasive means.

\section{Conclusion}

The use of the 3 salivary biomarkers, representing periodontal pathogen burden, inflammation, and tissue degradation, and calculating a cumulative risk score, based on these markers, reveal periodontitis more accurately than analyzing any of the salivary biomarkers alone.

\section{Acknowledgements}

The authors declare that they have no conflicts of interests. The present study is part of the Health 2000 Health Examination survey, organized by the National Institute for Health and Welfare of Finland, and financially supported by the Finnish Dental Association and the Finnish Dental Society. These series of studies were supported by grants from the IADR and the Orion, Paulo, Yrjö Jahnsson (grant 5639 for U. K. G) and Finnish Cultural Foundations (for U. K. G and grant 130408 for T. S.), the Academy of Finland (grant 78443 for E. K. and grant 118391 for P. J. P), the Sigrid Juselius Foundation (for P. J. P.) and the Helsinki University Central Hospital Research Foundation. Timo Sorsa is an inventor of US-patents 5652227, 5736341, 5866432 , and 6143476 .

\section{References}

[1] R.P. Darveau, Periodontitis: a polymicrobial disruption of host homeostasis, Nat Rev Microbiol 8 (2010), 481-490.

[2] B.L. Pihlström, Measurement of attachment level in clinical trials: probing methods, J Periodontol 63 (1992), 1072-1077.

[3] C.S. Miller, C.P. King, Jr., C. Langub, R.J. Krysio and M.V. Thomas, Salivary biomarkers of existing periodontal disease, J Am Dent Assoc 137 (2006), 322-329.

[4] C.S. Miller, J.D. Foley, A.L. Bailey, C.L. Campell, R.L. Humphries, N. Christodoulides, P.N. Floriano, G. Simmons, B. Bhagwandin, J.W. Jacobson, S.W. Redding, J.L. Ebersole and J.T. McDevitt, Current developments in salivary diagnostics, Biomark Med 4 (2010), 171-189.

[5] T. Sorsa, V.J. Uitto, K. Suomalainen, M. Vauhkonen and S. Lindy, Comparison of interstitial collagenases from human gingiva, sulcular fluid and polymorphonuclear leukocytes, $J$ Periodontal Res 23 (1988), 386-393.

[6] T. Sorsa, L. Tjäderhane and T. Salo, Matrix metalloproteinases (MMPs) in oral diseases, Oral Dis 10 (2004), 311-318.

[7] U.K. Gursoy, E. Könönen, P. Pradhan-Palikhe, T. Tervahartiala, P.J. Pussinen, L. Suominen-Taipale and T. Sorsa, Salivary MMP-8, TIMP-1, and ICTP as markers of advanced periodontitis, J Clin Periodontol 37 (2010), 487-493.

[8] M.Jr. Taba, J. Kinney, A.S. Kim and W.V. Giannobile, Diagnostic biomarkers for oral and periodontal diseases, Dent Clin North Am 49 (2005), 551-571.

[9] F.A. Scannapieco, P. Ng, K. Hovey, E. Hausmann and J. Wactawski-Wende, Salivary biomarkers associated with alveolar bone loss, Ann N Y Acad Sci 1098 (2007), 496-497.

[10] J.S. Kinney, C.A. Ramseier and W.V. Giannobile, Oral fluidbased biomarkers of alveolar bone loss in periodontitis, Ann N Y Acad Sci 1098 (2007), 230-251.

[11] S.I. Tobón-Arroyave, P.E. Jaramillo-González and D.M. IsazaGuzmán, Correlation between salivary IL-1 $\beta$ levels and periodontal clinical status, Arch Oral Biol 53 (2008), 346-352.

[12] E. Könönen, S. Paju, P.J. Pussinen, M. Hyvönen, P. Di Tella, L. Suominen-Taipale and M. Knuuttila, Population-based study of salivary carriage of periodontal pathogens in adults, $J$ Clin Microbiol 45 (2007), 2446-2451. 
[13] K. Hyvärinen, S. Laitinen, S. Paju, A.S. Hakala, L. SuominenTaipale, M. Skurnik, E. Könönen and P.J. Pussinen, Detection and quantification of five major periodontal pathogens by single copy gene-based real-time PCR, Innate Immun 15 (2009), 195-204.

[14] U.K. Gursoy, E. Könönen, V.J. Uitto, P. Pussinen, K. Hyvärinen, L. Suominen-Taipale and M. Knuuttila, Salivary interleukin- $1 \beta$ concentration and presence of multiple pathogens in periodontitis, J Clin Periodontol 36 (2009), 922 927.

[15] S. Paju, P.J. Pussinen, L. Suominen-Taipale, M. Hyvönen, M. Knuuttila and E. Könönen, Detection of multiple pathogenic species in saliva is associated with periodontal infection in adults, J Clin Microbiol 47 (2009), 235-238.

[16] B. Leblebicioglu, G. Kulekci, S. Ciftci, F. Keskin and S. Badur, Salivary detection of periodontopathic bacteria and periodontal health status in dental students, Anaerobe 15 (2009), 8286.

[17] Y. Sawamoto, N. Sugano, H. Tanaka and K. Ito, Detection of periodontopathic bacteria and an oxidative stress marker in saliva from periodontitis patients, Oral Microbiol Immunol $\mathbf{2 0}$ (2005), 216-220.

[18] S.M. Hamlet, M.P. Cullinan, B. Westerman, M. Lindeman, P.S. Bird, J. Palmer and G.J. Seymour, Distribution of Actinobacillus actinomycetemcomitans, Porphyromonas gingivalis and Prevotella intermedia in an Australian population, J Clin Periodontol 28 (2001), 1163-1171.

[19] R.P. Teles, V. Likhari, S.S. Socransky and A.D. Haffajee, Salivary cytokine levels in subjects with chronic periodontitis and in periodontally healthy individuals: a cross-sectional study, J Periodontal Res 44 (2009), 411-417.

[20] B.D. Frodge, J.L. Ebersole, R.J. Kryscio, M.V. Thomas and C.S. Miller, Bone remodeling biomarkers of periodontal disease in saliva, J Periodontol 79 (2008), 1913-1919.

[21] P.Y. Ng, M. Donley, E. Hausmann, A.D. Hutson, E.F. Rossomando and F.A. Scannapieco, Candidate salivary biomarkers associated with alveolar bone loss: cross-sectional and in vitro studies, FEMS Immunol Med Microbiol 49 (2007), 252-260.

[22] B. Zappacosta, S. Persichilli, A. Mordente, A. Minucci, D. Lazarro, E. Meucci and B. Giardina, Inhibition of salivary enzymes by cigarette smoke and the protective role of glutathione, Hum Exp Toxicol 21 (2002), 7-11.

[23] T. Sorsa, M. Hernandez, J. Leppilahti, S. Munjal, L. Neutschil and P. Mäntylä, Detection of gingival crevicular fluid MMP-8 levels with different laboratory and chair-side methods, Oral Dis 16 (2010), 39-45.

[24] J. Leppilahti, M.M. Ahonen, M. Hernandez, S. Munjal, L. Neutschil, V.J. Uitto, T. Sorsa and P. Mäntylä, Oral rinse MMP-8 point-of-care immuno tests identifies patients with strong periodontal inflammatory burden, Oral Dis 17 (2011), 115-122.

25] J.M. Goodson, A.C. Tanner, A.D. Haffajee, G.C. Sornberger and S.S. Socransky, Patterns of progression and regression of advanced destructive periodontal disease, J Clin Periodontol 9 (1982), 472-481.

[26] N. Silva, N. Dutzan, M. Hernandez, A. Dezerega, O. Rivera, J.C. Aguillon, O. Aravena, P. Lastres, P. Pozo, R. Vernal and J. Gamonal, Characterization of progressive periodontal lesions in chronic periodontitis patients: levels of chemokines, cytokines, matrix metalloproteinase-13, periodontal pathogens and inflammatory cells, J Clin Periodontol 35 (2008), 206214.

[27] S.S. Socransky and A.D. Haffajee, Periodontal microbial ecology, Periodontol 200038 (2005), 135-187.

[28] D.T. Graves and D. Cochran, The contribution of interleukin-1 and tumor necrosis factor to periodontal tissue destruction, $J$ Periodontol 74 (2003), 391-401.

[29] K.S. Kornman, R.C. Page, and M. S. Tonetti, The host response to the microbial challenge in periodontitis: assembling the players, Periodontol 200014 (1997), 33-53.

[30] P.J. Pussinen, S. Paju, P. Mäntylä and T. Sorsa, Serum microbial- and host-derived markers of periodontal diseases: a review, Curr Med Chem 14 (2007), 2402-2412.

[31] T. Nakashima, Y. Kobayashi, S. Yamasaki, A. Kawakami, K. Eguchi, H. Sasaki and H. Sakai, Protein expression and functional difference of membrane-bound and soluble receptor activator of NF-kappaB ligand: modulation of the expression by osteotropic factors and cytokines, Biochem Biophys Res Commun 275 (2000), 768-775.

[32] D.L. Cochran, Inflammation and bone loss in periodontal disease, J Periodontol 79 (2008), 1569-1576.

[33] C.A. Ramseier, J.S. Kinney, A.E. Herr, T. Braun, J.V. Sugai, C.A. Shelburne, L.A. Rayburn, H.M. Tran, A.K. Singh and W.V. Giannobile, Identification of pathogen and host-response markers correlated with periodontal disease, J Periodontol 80 (2009), 436-446.

[34] T. Sorsa, T. Ingman, K. Suomalainen, M. Haapasalo, Y.T. Konttinen, O. Lindy, H. Saari and V.J. Uitto, Identification of proteases from periodontopathogenic bacteria as activators of latent human neutrophil and fibroblast-type interstitial collagenases, Infect Immun 60 (1992), 4491-4495.

[35] T. Imamura, J. Travis, and J. Potempa, The biphasic virulence activities of gingipains: activation and inactivation of host proteins, Curr Protein Pept Sci 4 (2003), 443-450.

[36] P.G. Stathopoulou, M.R. Benakanakere, J.C. Galicia and D.F. Kinane, Epithelial cell pro-inflammatory cytokine response differs across dental plaque bacterial species, J Clin Periodontol 37 (2010), 24-29. 


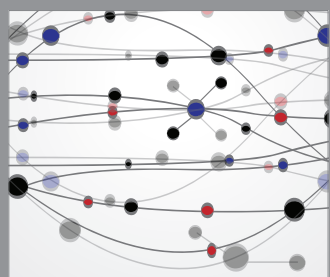

The Scientific World Journal
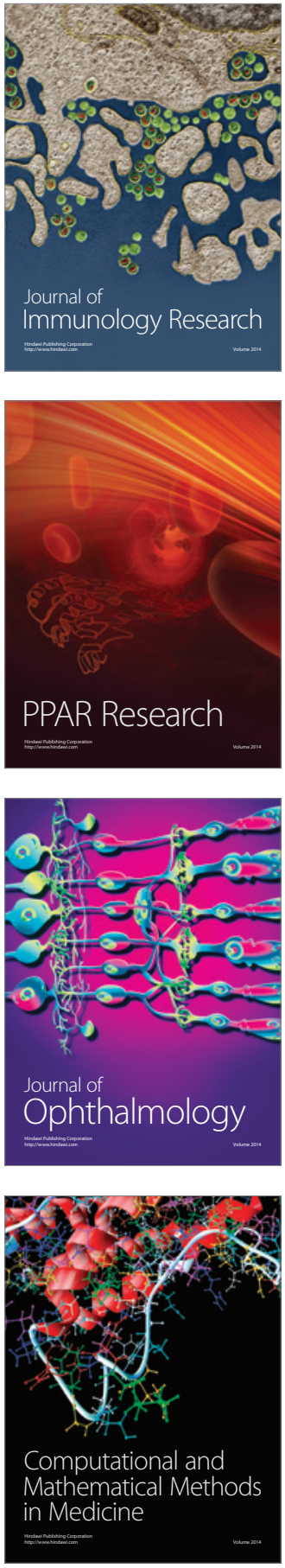

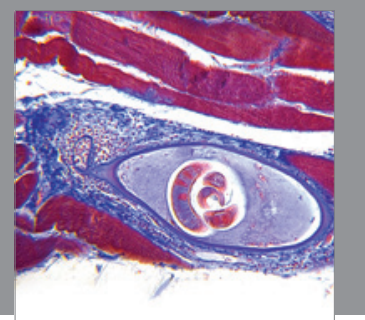

Gastroenterology

Research and Practice
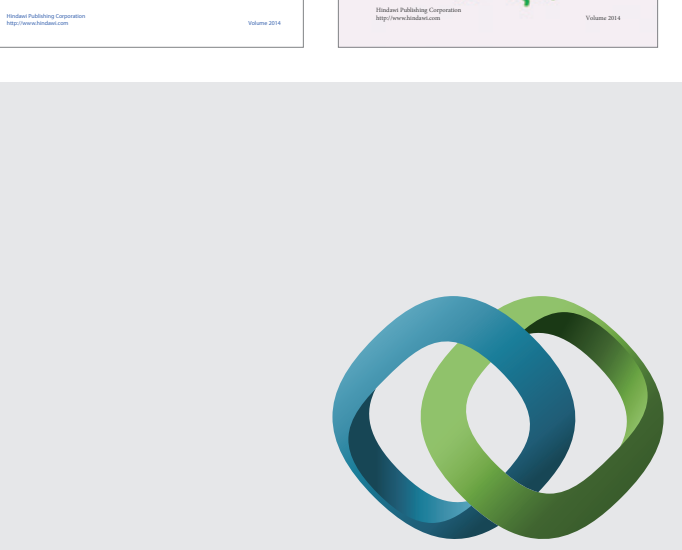

\section{Hindawi}

Submit your manuscripts at

http://www.hindawi.com
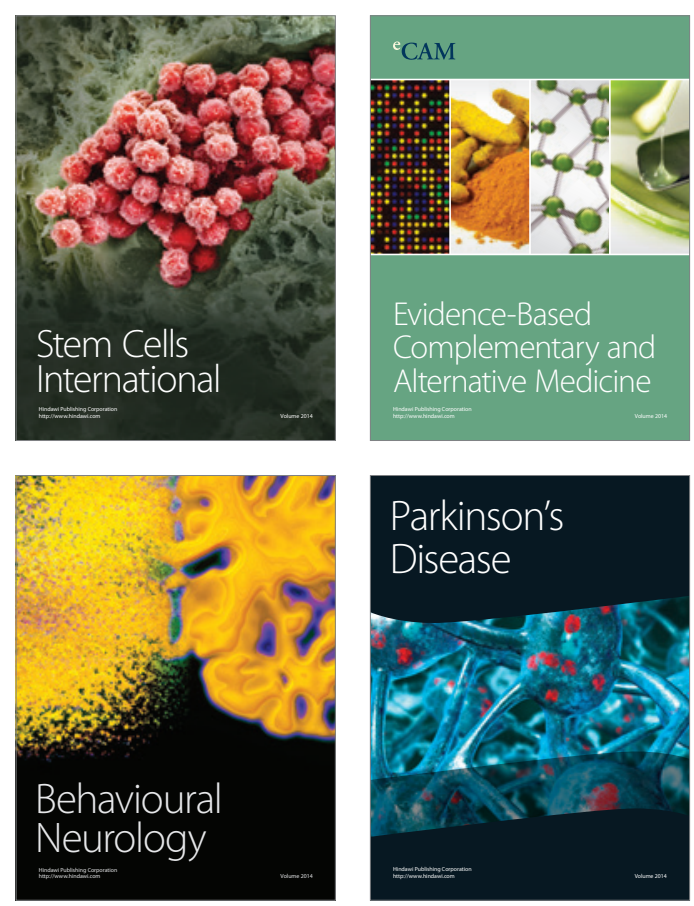

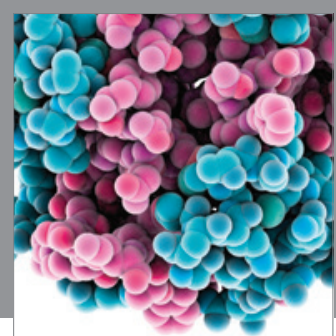

Journal of
Diabetes Research

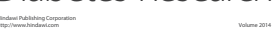

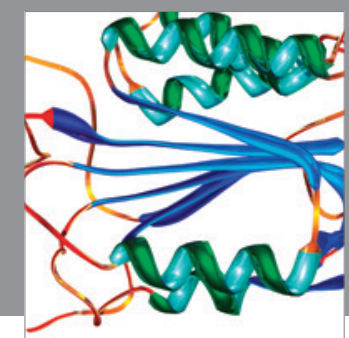

Disease Markers
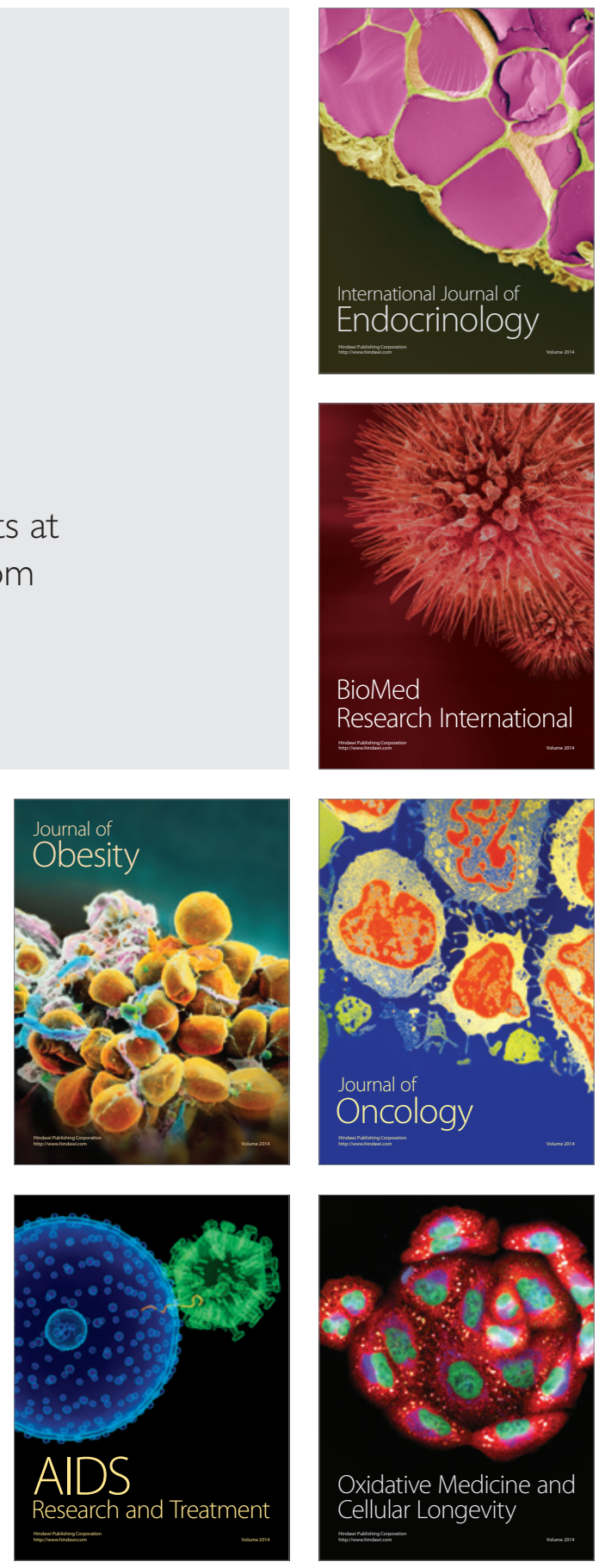